INTERNATIONAL JOURNAL OF MULTidisciplinARY RESEARCH AND ANALYSis

ISSN(print): 2643-9840, ISSN(online): 2643-9875

Volume 05 Issue 01 January 2022

DOI: 10.47191/ijmra/v5-i1-05, Impact Factor: 6.072

Page No.- 32-43

\title{
Stabiizing The Steady State Solution of Lasser Fever: Problems and Prospect
}

\author{
Innocent C. Eli ${ }^{1}$, Godspower C. Abanum ${ }^{2}$ \\ ${ }^{1}$ Department of Mathematics/Statistics, Ignatius Ajuru University of Education, Port Harcourt, Nigeria \\ ${ }^{2}$ Department of Mathematics/Statistics, Federal University Otuoke, Yenagoa, Nigeria
}

\begin{abstract}
The study of mathematical modeling of the stability analysis of Lassa fever was examined. A mathematical model for the spread and control of Lassa fever was formulated and analyzed. The model incorporates a control parameter, the use of condom to control human to human transmission through sexual contact with opposite sex. The disease free and endemic equilibrium states were analyzed.
\end{abstract}

KEYWORDS: Mathematical model, Ordinary differential equations, Stability, Laser Fever.

\section{INTRODUCTION}

Mathematical representation of disease parameters is a data reliant process and this prediction is often based on the implementation of mathematical models. The important feature is bridging the gap between mathematics (models) and the real world (data). In these models, the important features are theoretically developed but applied to real life scenarios represented by a given data. Such data may contain the signature of social effect, hence a comprehensive understanding of the phenomenon of disease involves a variety of mathematically tools, from model creation to the determination of solution to differential equation and to statistical analysis.

Lassa virus is a member of the Arenaviridae, a family of single-stranded RNA virus. This virus is an old world arenavirus which is enveloped, single-stranded and bi-segmented RNA. The natural reservoir of Lassa virus is multimammate Rat species known as mastomys natalensis which breed frequently and are distributed widely throughout West, Central and East Africa. They are the common rodents in Africa and they are found predominantly in rural areas and dwelling more often than surrounding country side. Member of this genus are infected persistently and shed the virus in their excrete and other bodily fluid throughout life. Sulaiman et al. (2018) investigated the transmission dynamics of the Lassa fever infection model with conclusion that the reproduction numbers in the humans are most sensitive to the transmission rates, recovery rates and the natural mortality rates of the humans, while the reproduction number in the rodents is most sensitive to the transmission rate, hunting/predation rate and the natural mortality rate of the rodents. The qualitative analysis of the study revealed that the disease becomes endemic in the rodents population and also do not die out of the human population over time without controlling the growth of the rodents population, preventing animal-humans transmissions and improvement on the recovery rates of humans.

Onuorah et al. (2016) studied the basic reproductive number for the spread and control of Lassa fever. In this research work, a mathematical model was developed and analyzed to study the transmission and control of Lassa fever. The model incorporate two control parameters, the use of rodenticide and condom. The disease free equilibrium points of the model were obtained and analyzed. They obtained an important threshold parameter called the basic reproductive number $R_{0}$, as it was known that when $R_{0}<1$ the disease dies out and when $R_{0}>1$ the disease persists in the population.

Lanlege et al. (2018) studied measuring the spread and control of Lassa fever using the basic reproduction number $R_{0}$. In this study, a mathematical model for the spread and control of Lassa fever was presented showing that the most effective strategy to curb the disease is the usage of condom. It was evaluated that as the compliance to the use of condom as a control measure increase, the reproduction number $R_{0}$ decreases to less than one $\left(R_{0}<1\right)$ which means that the disease is under control within the country and when the control measure is at lower rate or moderate rate then $R_{0}$, hence the disease is not under control within the country. 


\section{Stabiizing The Steady State Solution of Lasser Fever: Problems and Prospect}

A mathematical model of the transmission dynamics of Lassa fever with separation of infected individual and treatment as control measures was studied by Nwasuka et al. (2019). The model assumes susceptible humans acquired the infection through interaction with the infected rodent populations. It was found that treatment is only given to separate human population. $A$ threshold parameter called the effective reproduction number $R_{\text {eff }}$ was obtained. The disease free equilibrium state was obtained and analyzed before the reproduction number was obtained. If $R_{e f f}<1$ the disease-free equilibrium exists and it is locally and globally asymptotically stable, implying that Lassa fever can be controlled and eradicated within the population in a finite time and if the

$R_{e f f}>1$, the disease invade and become endemic in the population.

A study by Eraikhueman et al. (2017) considered Lassa fever and its control measures. In their study, a mathematical model was used to investigate the dynamics of spread of Lassa fever in human population. The contributions from regular contact with the species of rodents that carry the virus that cause Lassa fever and infections, contract with persons suffering from the disease was examined to be significant in the spread of the disease. The paper gathered among several intervention measures, control of the rodents carrying the virus, isolation policy for persons infected with the virus and introducing vaccines to the human population are some of the best strategies against the spread of the disease.

Obabiyi et al. (2017) discussed a mathematical model for Lassa fever transmission dynamics with variable human and reservoir population. Their study highlighted a compartmental modeling approach for two different populations: human and rodent with rigorous analysis. The analytical results showed the spread of Lassa fever can be effectively controlled in the population if the associated intervention strategies can make $R_{0}$ less than $1\left(R_{0}<1\right)$. But if $R_{0}>1$ the disease will persist in the population. The results of simulations reveal that increase in recovery rate contributes to decrease in the number of exposed and infectious human in the population and increase in the number of recovered human. The study concluded that early diagnostic of infected humans, maintaining hygienic environment, use of new needle when taking injection and interim control of the rodent carrying the virus are the best strategies against the spread of the disease.

Akinwande et al. (2018) investigated a mathematical model for Lassa fever and sensitivity analysis. In this study, a mathematical model of five compartments was formulated. The disease free equilibrium point of the model was performed and the local and global stability was obtained and analyzed based on the reproduction number. The sensitivity analysis shows that the most sensitive parameters to the basic reproduction number $R_{0}$ are the contact rate $\beta$

Faniran (2017) investigated a mathematical modeling of Lassa fever dynamics with non-drug compliance rate. The model incorporate a non-drug compliance rate in the parameters for the human population. The basic reproduction number was derived and so also was the stability of the disease-free and endemic equilibrium points analyzed. It was established that the disease-free equilibrium is globally asymptotically stable when the reproduction, $R_{0}<1$ and the disease always dies out. Also, the diseasefree equilibrium point becomes unstable when $R_{0}>1$, hence endemic equilibrium point is globally asymptotically stable.

Sensitivity analysis of Lassa fever model by Onuorah et al. (2016), an extension of the proposed model by Onuorah and Akinwande was formulated by incorporating the sensitivity analysis. Sensitivity tells us how important each parameter is to disease transmission. Key to analysis was the reproduction number. Reasonable sets of values for the parameter in the model were compiled and sensitivity analysis around the baseline parameter value were computed, which shows that the most sensitive parameter to $R_{0}$ is human birth rate, followed by condom efficacy and compliance. Obasi et al. estimated the basic reproduction of Lassa fever epidemics and its relationship with inter-epidemic period. (2019). In this work, a realistic mathematical model that incorporate all the known transmission routes into Lassa fever epidemiology was presented. The basic reproduction number for the model $R_{0}$, was derived and the inter-epidemic period of infection disease was examined. From the result, it can be said that the inter-epidemic period is useful to estimate the effect of intervention on Lassa fever incidence quantitatively. The paper highlighted the relationship between the Lassa fever basic reproduction and inter-epidemic period of infection disease. Therefore, any policy or measures taken to reduce the basic reproduction number will definitely make the disease to take a very long time to re-occur.

Akinade et al. (2020) studied sensitivity and stability analysis of a Lassa fever disease model with control strategies. The research brought into limelight three control strategies which include; early diagnosis of the the infected human before onset of noticeable symptoms resulting in the treatment of the human-carriers, vaccination of the susceptible humans and the use of rodenticide on the vector populations as effective means of limiting the spread of Lassa fever disease within an endemic population. An increase in the value of these three controls, yields a rapid and continuous decrease in the members of the carrier human, infected human and vector populations and eventually in the eradication of the disease from an endemic population. In this work, the recovered population experiences a continuous increase and no decrease in its size suggesting that these controls are very efficacious in 


\section{Stabiizing The Steady State Solution of Lasser Fever: Problems and Prospect}

controlling the disease. The basic reproduction number was obtained, so also was the disease-free equilibrium analyzed for stability. The sensitivity analysis results show the sensitiveness of each model parameter to the transmission of the disease and it was obtained that the rate at which the susceptible human population contracts the infection via contact with human-carrier is the most sensitive parameter to the spread of the disease.

Sensitivity analysis of multiple control intervention measures of Lassa fever disease model by Onah et al. (2019) was developed that incorporates the basic and important dynamics of Lassa fever disease transmission under the assumption of a homogeneously mixed population. The study extended the model by introducing various control intervention measures, like external protection, isolation, treatment and rodent control. Sensitivity analysis was carried out to investigate the impact of the control parameters on the persistence or eradication of Lassa fever disease.

\section{MODEL FORMULATION}

For the sake of clarity in this chapter, we assume a homogeneous mixing of the human and vector population such that there are equal chances of transmitting the virus when there is contact between susceptible human and active vector. Thus the total human population at time $\mathrm{t}$ denoted by $\mathrm{N}_{H}(\mathrm{t})$ is sub-divided into four (4) and they are; susceptible male $\mathrm{S}_{M}(\mathrm{t})$, infected male $\mathrm{I}_{M}(\mathrm{t})$, susceptible female $S_{F}(t)$, infected female $I_{F}(t)$ Hence:

$$
\mathrm{N}_{H}(\mathrm{t})=\mathrm{S}_{M}(\mathrm{t})+\mathrm{I}_{M}(\mathrm{t})+\mathrm{S}_{F}(\mathrm{t})+\mathrm{I}_{F}(\mathrm{t})
$$

Similarly, the total vector population at timet, denoted by $\mathrm{N}_{V}(\mathrm{t})$ is sub-divided into dormant vector $\mathrm{D}_{V}(\mathrm{t})$ and active vector $\mathrm{A}_{V}(\mathrm{t})$ , such that

$$
\mathrm{N}_{V}(\mathrm{t})=\mathrm{D}_{V}(\mathrm{t})+\mathrm{A}_{V}(\mathrm{t})
$$

The schematic diagram of the model is shown in fig 3.1 .

The dashed line from infected male $\mathrm{I}_{M}$ to susceptible female $\mathrm{S}_{F}$ shows that the infected male infects the susceptible female. This could be as a result of sexual interaction between the infected male and susceptible female.

The line from infected female $I_{F}$ to susceptible male $S_{M}$ shows that the infected female infects the susceptible male. This also could be as a result of sexual interaction between the infected female and susceptible male.

The dashed line also from active vector $\mathrm{A}_{V}$ (carrier vector) to the susceptible male population $\mathrm{S}_{M}$ shows the transfer of the virus from infected rodent to susceptible male. When susceptible male is exposed to the droppings of infected mastomy rats, they become infected.

The dashed line from active vector $\mathrm{A}_{V}$ (carrier vector) to the susceptible female population $\mathrm{S}_{F}$ shows the transfer of the virus from infected rodent to susceptible female. That is, susceptible female become infected with Lassa fever virus from exposure to the droppings of infected mastomy rats.

The susceptible male population $\mathrm{S}_{M}(\mathrm{t})$ is generated through male birth at the rate $\beta_{H} \theta \mathrm{N}_{H}$ where $0<\theta<1$.

The dormant vector $\mathrm{D}_{V}$ population is generated through natural birth rate by active vector $\mathrm{A}_{V}$ denoted by $\beta_{R} \mathrm{~N}_{V}$ and is reduced by progression to active vector $\left(\mathrm{A}_{V}\right)$ population due to maturity at the rate $\sigma$, natural death at the rate $\mu_{2}$ and death due to application of pesticide at the rate $\delta_{2}$ Males who recovered from the infection at the rate $\gamma$ is at the other hand reduced by natural death at the rate $\mu_{1}$, interaction with infected female $\mathrm{I}_{F}$ and active vector $\mathrm{A}_{V}$, which results to the force of infection.

$$
\text { i. e; } \frac{\left(C_{2} \alpha_{1}(1-\varepsilon \tau) I_{F}+\alpha_{3} A_{V}\right) S_{M}}{N_{H}} \text {, where }
$$

$\mathrm{C}_{2}$ is the average sexual partners acquired by susceptible male $\mathrm{S}_{M}$

$\alpha_{1}$ is the probability of transmission of infection resulting from sexual interaction between infected infected female and susceptible male.

The factor $(1-\varepsilon \tau)$ is the effect of condom usage by susceptible male $S_{M}$.

$\alpha_{3}$ is the probability of transmission of infection when there is interaction between Active Vector and Susceptible male.

The infected male population $I_{M}(t)$ is generated by sexual interaction with infected female $I_{F}(t)$ and interaction with Active vector $A_{V}(t)$ i.e

$$
\frac{\left(C_{2} \alpha_{1}(1-\varepsilon \tau) I_{F}+\alpha_{3} A_{V}\right) S_{M}}{N_{H}} .
$$

It is decreased by natural death at the rate $\mu_{1}$, death due to infection at the rate $\delta_{1}$ and recovery of infected male $I_{M}$ as a result of treatment at the rate $\gamma$. The Susceptible female $S_{F}(t)$ population is generated through female birth at the rate $\beta_{H}(1-\theta) N_{H}$ where

$0<\theta<1$. It is increased by recovered infected female at the rate $\gamma$. It is on the other hand reduced by natural death at the rate $\mu_{1}$ and interaction with infected male $I_{M}$ and Active Vector $A_{V}$ which results to the force of infection $\frac{\left(C_{1} \alpha_{2}(1-\varepsilon \tau) I_{M}+\alpha_{4} A_{V}\right) S_{F}}{N_{H}}$ where $C_{1}$ is the average sexual partners acquired by susceptible females. 


\section{Stabiizing The Steady State Solution of Lasser Fever: Problems and Prospect}

$\alpha_{2}$ is the probability of transmission of infection when there is interaction between infected male $I_{M}$ and Susceptible female. The factor $(1-\tau \varepsilon)$ is the effect of condom usage by Susceptible female.

$\alpha_{4}$ is the probability of transmission of infection when there is interaction between Active Vector and Susceptible female.

The infected female $I_{F}(t)$ population is generated by sexual interaction with infected male $I_{M}$ and interaction with Active Vector $A_{V}$ i.e

$\frac{\left(C_{1} \alpha_{2}(1-\varepsilon \tau) I_{M}+\alpha_{4} A_{V}\right) S_{F}}{N_{H}}$. It is decreased by natural death at the rate $\mu_{1}$, death due to infection at the rate $\delta_{1}$ and recovery of infected female $I_{F}$ as a result of treatment at the rate $\gamma$.

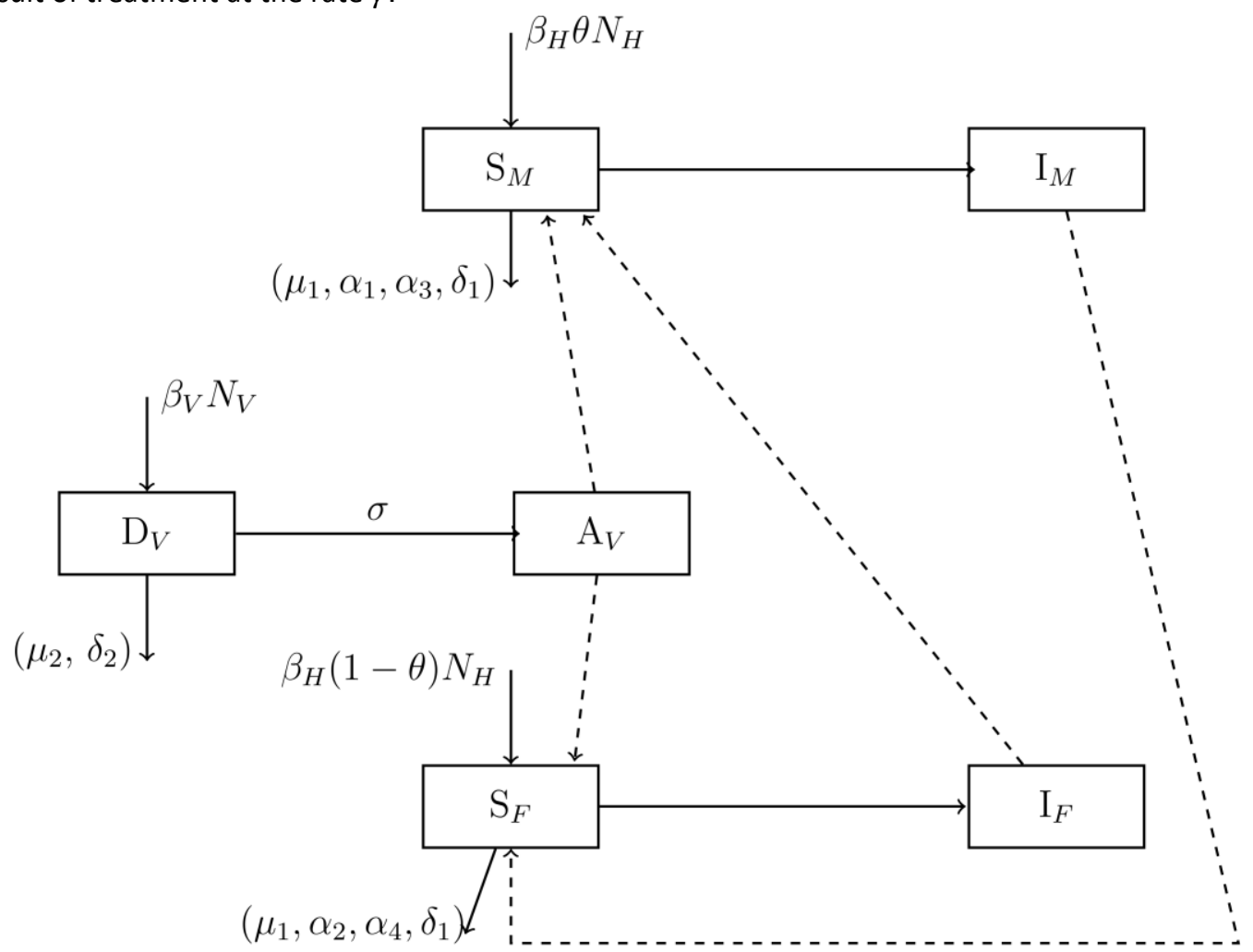

Figure3. 1: Schematic Diagram of the Mathematical model for the Lassa Fever Transmission.

\section{MODEL ASSUMPTIONS}

We assumed that:

1. New births are Susceptible

2. Virus does not kill the reservoir host. I.e. their death can be natural or accidental.

3. Vector class is divided into two; Active Vector $A_{V}$ and Dormant Vector $D_{V}$

\section{MODEL EQUATIONS}

Considering the above assumptions, definition of variables and parameters and relationship between the variables and parameters described in the schematic diagram, we developed six (6) ordinary differential equations for the causes, spread and control of Lassa fever in a population. The differential equations are given as the following;

$$
\begin{aligned}
& \frac{d S_{M}}{d T}=\beta H \theta N_{H}+\gamma I_{M}-\frac{\left(C_{2} \alpha_{1}(1-\varepsilon \tau) I_{F}+\alpha_{3} A_{V}\right) S_{M}}{N_{H}}-\mu_{1} S_{M} . \\
& \frac{d I_{M}}{d T}=\frac{\left(C_{2} \alpha_{1}(1-\varepsilon \tau) I_{F}+\alpha_{3} A_{V}\right) S_{M}}{N_{H}}-\left(\mu_{1}+\delta_{1}+\gamma\right) I_{M} . \\
& \frac{d S_{F}}{d T}=\beta H(1-\theta) N_{H}+\gamma I_{F}-\frac{\left(C_{1} \alpha_{2}(1-\varepsilon \tau) I_{M}+\alpha_{4} A_{V}\right) S_{F}}{N_{H}}-\mu_{1} S_{F} \\
& \frac{d I_{F}}{d T}=\frac{\left(C_{1} \alpha_{2}(1-\epsilon \tau) I_{M}+\alpha_{4} A_{V}\right) S_{F}}{N_{H}}-\left(\mu_{1}+\delta_{1}+\gamma\right) I_{F} \\
& \frac{d D_{V}}{d T}=\beta_{V} N_{V}-\left(\sigma+\mu_{2}+\delta_{2}\right) D_{V}
\end{aligned}
$$




$$
\frac{d A_{V}}{d T}=\sigma D_{V}-\left(\mu_{2}+\delta_{2}\right) A_{V}
$$

\section{Where}

\begin{tabular}{|c|c|}
\hline$S_{M}(t)$ & Number of susceptible male at time $\mathbf{t}$ \\
\hline$I_{M}(t)$ & Number of Infected male at time $\mathbf{t}$ \\
\hline$S_{F}(t)$ & Number of susceptible female at time $\mathbf{t}$ \\
\hline$I_{F}(t)$ & Number of Infected female at time $\mathbf{t}$ \\
\hline$D_{V}(t)$ & Number of dormant vector at time $\mathbf{t}$ \\
\hline$A_{V}(t)$ & Number of active vector at time $\mathbf{t}$ \\
\hline$\beta_{H}$ & The natural birth rate of Human population \\
\hline$\beta_{V}$ & The natural birth rate of Vectors \\
\hline$\theta$ & The proportion of Human birth that is male $(0<\theta<1)$ \\
\hline$\alpha_{1}$ & $\begin{array}{l}\text { The probability of transmission of infection resulting from } \\
\text { sexual interaction between infected female and susceptible } \\
\text { male }\end{array}$ \\
\hline$\alpha_{2}$ & $\begin{array}{l}\text { The probability of transmission of infection resulting from } \\
\text { sexual interaction between infected male and susceptible } \\
\text { female }\end{array}$ \\
\hline$\alpha_{3}$ & $\begin{array}{l}\text { The probability of transmission of infection resulting from } \\
\text { interaction between active vectors and susceptible male }\end{array}$ \\
\hline$\alpha_{4}$ & $\begin{array}{l}\text { The probability of transmission of infection resulting from } \\
\text { interaction between active vectors and susceptible female }\end{array}$ \\
\hline$c_{1}$ & $\begin{array}{l}\text { Average number of male partners acquired by a susceptible } \\
\text { female }\end{array}$ \\
\hline$c_{2}$ & $\begin{array}{l}\text { Average number of male partners acquired by a susceptible } \\
\text { male }\end{array}$ \\
\hline$\mu_{1}$ & Natural death rate of Human population \\
\hline$\mu_{2}$ & Natural death rate of vector population \\
\hline$\sigma$ & Progression rate from dormant to active vector \\
\hline$\delta_{1}$ & Death rate of Human population due to infection \\
\hline$\delta_{2}$ & Death rate of vector population due to application of pesticide \\
\hline$\gamma$ & Recovery rate of infected Human \\
\hline$\varepsilon$ & Efficacy of condom \\
\hline$\tau$ & Compliance of condom usage \\
\hline
\end{tabular}

From the above model equations, by adding equations (1)--(4) we can derive the rate of change of the total Human population. Thus;

$$
\begin{aligned}
& \beta_{H} \theta N_{H}+\gamma I_{M}-\frac{\left(C_{2} \alpha_{1}(1-\varepsilon \tau) I_{F}+\alpha_{3} A_{V}\right) S_{M}}{N_{H}}-\mu_{1} S_{M}+\frac{\left(C_{2} \alpha_{1}(1-\varepsilon \tau) I_{F}+\alpha_{3} A_{V}\right) S_{M}}{N_{H}}-\left(\mu_{1}+\delta_{1}+\gamma\right) I_{M}+\beta_{H}(1-\theta) N_{H}+\gamma I_{F} \\
& \\
& \quad-\frac{\left(C_{1} \alpha_{2}(1-\varepsilon \tau) I_{M}+\alpha_{4} A_{V}\right) S_{F}}{N_{H}}-\mu_{1} S_{F}+\frac{\left(C_{1} \alpha_{2}(1-\varepsilon \tau) I_{M}+\alpha_{4} A V\right) S_{F}}{N_{H}}-\left(\mu_{1}+\delta_{1}+\gamma\right) I_{F} \\
& =\beta_{H} \theta N_{H}+\gamma I_{M}-\mu_{1} S_{M}-\left(\mu_{1}+\delta_{1}+\gamma\right) I_{M}+\beta_{H}(1-\theta) N_{H}+\gamma I_{F}-\mu_{1} S_{F}-\left(\mu_{1}+\delta_{1}+\gamma\right) I_{F} \\
& =\beta H \theta N_{H}+\gamma I_{M}-\mu_{1} S_{M}-\left(\mu_{1}+\delta_{1}\right) I_{M}-\gamma I_{M}+\beta_{H}(1-\theta) N_{H}+\gamma I_{F}-\mu_{1} S_{F}-\left(\mu_{1}+\delta_{1}\right) I_{F}-\gamma I_{F} \\
& =\beta_{H} \theta N_{H}-\mu_{1} S_{M}-\left(\mu_{1}+\delta_{1}\right) I_{M}+\beta_{H}(1-\theta) N_{H}-\mu_{1} S_{F}-\left(\mu_{1}+\delta_{1}\right) I_{F} \\
& \delta_{1} I_{M}+\beta_{H} N_{H}-\beta_{H} \theta N_{H}-\mu_{1} S_{F}-\mu_{1} I_{F}-\delta_{1} I_{F} \theta N_{H}-\mu_{1} S_{M}-\mu_{1} I_{M}- \\
& =\beta_{H} N_{H}-\mu_{1}\left(S_{M}+I_{M}+S_{F}+I_{F}\right)-\delta_{1}\left(I_{M}+I_{F}\right) \quad=\beta_{H} N_{H}-\mu_{1} S_{M}-\mu_{1} I_{M}-\mu_{1} S_{F}-\mu_{1} I_{F}-\delta_{1} I_{M}-\delta_{1} I_{F}
\end{aligned}
$$

Recall that

Hence;

$$
N_{H}=S_{M}+I_{M}+S_{F}+I_{F}
$$

$$
\beta_{H} N_{H}-\mu_{1} N_{H}-\delta_{1}\left(I_{M}+I_{F}\right)
$$

Hence, the rate of change of the total human population is 


$$
\frac{d N_{H}}{d T}=\beta_{H} N_{H}-\mu_{1} N_{H}-\delta_{1}\left(I_{M}+I_{F}\right)
$$

Also by adding equations (5)-(6), we can derive the rate of change of the total vector population. Thus;

$=\beta_{V} N_{V}-\left(\sigma+\mu_{2}+\delta_{2}\right) D_{V}+\sigma D_{V}-\left(\mu_{2}+\delta_{2}\right) A_{V}$

$=\beta_{V} N_{V}-\sigma D_{V}-\mu_{2} D_{V}-\delta_{2} D_{V}+\sigma D_{V}-\mu_{2} A_{V}-\delta_{2} A_{V}$

$=\beta_{V} N_{V}-\mu_{2} D_{V}-\delta_{2} D_{V}-\mu_{2} A_{V}-\delta_{2} A_{V}$

$=\beta_{V} N_{V}-D_{V}\left(\mu_{2}+\delta_{2}\right)-A_{V}\left(\mu_{2}+\delta_{2}\right)$

$=\beta_{V} N_{V}-\left(\mu_{2}+\delta_{2}\right)\left(D_{V}+A_{V}\right)$

Recall that $N_{V}=D_{V}+A_{V}$

Hence; $=\beta_{V} N_{V}-\left(\mu_{2}+\delta_{2}\right) N_{V}$

Therefore the rate of change of the total vector population is

$$
\frac{d N_{V}}{d T}=\beta_{V} N_{V}-\left(\mu_{2}+\delta_{2}\right) N_{V}
$$

\section{EQUILIBRIUM STATE}

Using the dictionary definition of equilibrium, we know it to be a state of balance. But with respect to this chapter, we will narrow down the definition as, at equilibrium state, the rate of change must be equal to zero (0). i.e.

We let;

$$
\frac{d S_{M}}{d T}=\frac{d I_{M}}{d T}=\frac{d S_{F}}{d T}=\frac{d I_{F}}{d T}=\frac{d D_{V}}{d T}=\frac{d A_{V}}{d T}=0
$$

$$
\left(S_{M}, I_{M}, S_{F}, I_{F}, D_{V}, A_{V}\right)=(X, Y, Z, U, V, W)
$$

\section{DISEASE-FREE EQUILIBRIUM STATE}

From the name, we derive the definition. It is a state in which a disease does not exist. So from this we have that the classes where the disease does not exist equals zero (0) i.e. at the disease-free equilibrium state;

$$
I_{M}=I_{F}=D_{V}=A_{V}=0
$$

Substitute equation (3.9) into equation (3.1)

Making $S_{M}$ subject formulae

$$
\begin{gathered}
\beta_{H} \theta N_{H}+\gamma I_{M}-\frac{\left(C_{2} \alpha_{1}(1-\varepsilon \tau) I_{F}+\alpha_{3} A_{V}\right) S_{M}}{N_{H}}-\mu_{1} S_{M}=0 \\
\beta H \theta N_{H}+\gamma(0)-\frac{\left(C_{2} \alpha_{1}(1-\varepsilon \tau)(0)+\alpha_{3}(0)\right) S_{M}}{N_{H}}-\mu_{1} S_{M}=0 \\
\beta_{H} \theta N_{H}-\mu_{1} S_{M}=0 \Rightarrow \mu_{1} S_{M}=\beta_{H} \theta N_{H}
\end{gathered}
$$

$$
S_{M}=\frac{\beta_{H} \theta N_{H}}{\mu_{1}}
$$

Also substituting equation (3.9) into equation (3.3)

\section{Where}

$$
\begin{gathered}
\frac{d S_{F}}{d T}=\beta_{H}(1-\theta) N_{H}+\gamma I_{F}-\frac{\left(C_{1} \alpha_{2}(1-\varepsilon \tau) I_{M}+\alpha_{4} A_{V}\right) S_{F}}{N_{H}}-\mu_{1} S_{F} \\
\beta_{H}(1-\theta) N_{H}+\gamma I_{F}-\frac{\left(C_{1} \alpha_{2}(1-\varepsilon \tau) I_{M}+\alpha_{4} A_{V}\right) S_{F}}{N_{H}}-\mu_{1} S_{F}=0 \\
\beta H(1-\theta) N_{H}+\gamma(0)-\frac{\left(C_{1} \alpha_{2}(1-\varepsilon \tau)(0)+\alpha_{4}(0)\right) S_{F}}{N_{H}}-\mu_{1} S_{F}=0 \\
\quad \beta H(1-\theta) N_{H}-\mu_{1} S_{F}=0 \Rightarrow \mu_{1} S_{F}=\beta_{H}(1-\theta) N_{H} \\
S_{F}=\frac{\beta_{H}(1-\theta) N_{H}}{\mu_{1}}
\end{gathered}
$$

Hence the disease free equilibrium (DFE) state is

$$
\left(S_{M}, I_{M}, S_{F}, I_{F}, D_{V}, A_{V}\right)=(X, Y, Z, U, V, W)
$$

$$
E_{0}=(X, \mathrm{Y}, Z, U, V, W)=\left[\frac{\beta_{H} \theta N_{H}}{\mu_{1}}, 0, \frac{\beta_{H} H(1-\theta) N_{H}}{\mu_{1}}, 0,0,0\right]
$$

\section{ENDEMIC EQUILIBRIUM STATE}

In the endemic state, the disease exists for every class in the population. That is the endemic equilibrium state is the state where the disease cannot be totally eradicated but remains in the population. Hence; 


\section{Stabiizing The Steady State Solution of Lasser Fever: Problems and Prospect}

$$
I_{M}=I_{F}=D_{V}=A_{V} \neq 0
$$

To obtain the number of susceptible male $S_{M}$ at the endemic equilibrium state, we solve equation (3.2)

$$
\begin{gathered}
\frac{d I_{M}}{d T}=\frac{\left(C_{2} \alpha_{1}(1-\varepsilon \tau) I_{F}+\alpha_{3} A_{V}\right) S_{M}}{N_{H}}-\left(\mu_{1}+\delta_{1}+\gamma\right) I_{M} \\
0=\frac{\left(C_{2} \alpha_{1}(1-\varepsilon \tau) I_{F}+\alpha_{3} A_{V}\right) S_{M}}{N_{H}}-\left(\mu_{1}+\delta_{1}+\gamma\right) I_{M} \\
\frac{\left(C_{2} \alpha_{1}(1-\varepsilon \tau) I_{F}+\alpha_{3} A_{V}\right) S_{M}}{N_{H}}=\left(\mu_{1}+\delta_{1}+\gamma\right) I_{M} \\
S_{M}=\frac{N_{H}\left(\mu_{1}+\delta_{1}+\gamma\right) I_{M}}{\left(C_{2} \alpha_{1}(1-\varepsilon \tau) I_{F}+\alpha_{3} A_{V}\right)}=\frac{N_{H}\left(\mu_{1}+\delta_{1}+\gamma\right) I_{M}}{C_{2} \alpha_{1}(1-\varepsilon \tau) I_{F}+\alpha_{3} A_{V}}
\end{gathered}
$$

To obtain the number of infected male $I_{M}$ at the endemic equilibrium state, we solve equation (1)

$$
\begin{gathered}
\frac{d S_{M}}{d T}=\beta H \theta N_{H}+\gamma I_{M}-\frac{\left(C_{2} \alpha_{1}(1-\varepsilon \tau) I_{F}+\alpha_{3} A_{V}\right) S_{M}}{N_{H}}-\mu_{1} S_{M} . \\
0=\beta_{H} \theta N_{H}+\gamma I_{M}-\frac{\left(C_{2} \alpha_{1}(1-\varepsilon \tau) I_{F}+\alpha_{3} A_{V}\right) S_{M}}{N_{H}}-\mu_{1} S_{M} . \\
S_{M}\left[\frac{C_{2} \alpha_{1}(1-\varepsilon \tau) I_{F}+\alpha_{3} A_{V}}{N_{H}}+\mu_{1}\right]=\beta_{H} \theta N_{H}+\gamma I_{M} \\
S_{M}\left[\frac{C_{2} \alpha_{1}(1-\varepsilon \tau) I_{F}+\alpha_{3} A_{V}+N_{H} \mu_{1}}{N_{H}}\right]=\beta_{H} \theta N_{H}+\gamma I_{M} \\
S_{M}=\frac{N_{H}\left(\beta_{H} \theta N_{H}+\gamma I_{M}\right)}{C_{2} \alpha_{1}(1-\varepsilon \tau) I_{F}+\alpha_{3} A_{V}+N_{H} \mu_{1}}
\end{gathered}
$$

Substituting equation (3.13) into (3.14)

$$
\begin{aligned}
& \frac{N_{H}\left(\mu_{1}+\delta_{1}+\gamma\right) I_{M}}{C_{2} \alpha_{1}(1-\varepsilon \tau) I_{F}+\alpha_{3} A_{V}}= \frac{N_{H}\left(\beta_{H} \theta N_{H}+\gamma I_{M}\right)}{C_{2} \alpha_{1}(1-\xi j \tau) I_{F}+\alpha_{3} A_{V}+N_{H} \mu_{1}} \\
& \frac{\left(\mu_{1}+\delta_{1}+\gamma\right) I_{M}}{C_{2} \alpha_{1}(1-\varepsilon \tau) I_{F}+\alpha_{3} A_{V}}= \frac{\left(\beta_{H} \theta N_{H}+\gamma I_{M}\right)}{C_{2} \alpha_{1}(1-\varepsilon \tau) I_{F}+\alpha_{3} A_{V}+N_{H} \mu_{1}} \\
& \text { Let } C_{2} \alpha_{1}(1-\varepsilon \tau)=P \text { and }\left(\mu_{1}+\delta_{1}+\gamma\right)=A \\
& \frac{A I_{M}}{P I_{F}+\alpha_{3} A_{V}}= \frac{\beta_{H} \theta N_{H}+\gamma I_{M}}{P I_{F}+\alpha_{3} A_{V}+N_{H}+\mu_{1}} \\
& A I_{M} P I_{F}+A I_{M} \alpha_{3} A_{V}+A I_{M} N_{H} \mu_{1}=P I_{F} \beta_{H} \theta N_{H}+P I_{F} \gamma I_{M}+\alpha_{3} A_{V H} \beta_{H} \theta N_{H}+\alpha_{3} A_{V} \gamma I_{M}
\end{aligned}
$$

Collect like terms

$$
\begin{gathered}
A I_{M} P I_{F}+A I_{M} \alpha_{3} A_{V}+A I_{M} N_{H} \mu_{1}-P I_{F} \gamma I_{M}-\alpha_{3} A_{V} \gamma I_{M}=P I_{F} \beta H \theta N_{H}+\alpha_{3} A_{V} \beta_{H} \theta N_{H} \\
I_{M}\left(P A I_{F}+A \alpha_{3} A_{V}+A N_{H} \mu_{1}-P I_{F} \gamma-\alpha_{3} A_{V} \gamma\right)=P I_{F} \beta_{H} \theta N_{H}+\alpha_{3} A_{V} \beta_{H} \theta N_{H} \\
I_{M}=\frac{P I_{F} \beta H \theta N_{H}+\alpha_{3} A_{V} \beta_{H} \theta N_{H}}{P A I_{F}+A \alpha_{3} A_{V}+A N_{H} \mu_{1}-P I_{F} \gamma-\alpha_{3} A_{V} \gamma}
\end{gathered}
$$

Thus

$$
I_{M}=\frac{\beta_{H} \theta N_{H}\left[C_{2} \alpha_{1}(1-\epsilon \tau) I_{F}+\alpha_{3} A_{V}\right]}{\left(\mu_{1}+\delta_{1}+\gamma\right)\left[C_{2} \alpha_{1}(1-\varepsilon \tau) I_{F}+\alpha_{3} A_{V}+N_{H} \mu_{1}\right]-C_{2} \alpha_{1}(1-\varepsilon \tau) I_{F} \gamma-\alpha_{3} A_{V} \gamma}
$$

To solve for the number of susceptible female $S_{F}$ at the endemic equilibrium state, we solve equation (4)

$$
\begin{gathered}
\frac{d I_{F}}{d T}=\frac{\left(C_{1} \alpha_{2}(1-\varepsilon \tau) I_{M}+\alpha_{4} A_{V}\right) S_{F}}{N_{H}}-\left(\mu_{1}+\delta_{1}+\gamma\right) I_{F} \\
0=\frac{\left(C_{1} \alpha_{2}(1-\varepsilon \tau) I_{M}+\alpha_{4} A_{V}\right) S_{F}}{N_{H}}-\left(\mu_{1}+\delta_{1}+\gamma\right) I_{F} \\
\frac{\left(C_{1} \alpha_{2}(1-\varepsilon \tau) I_{M}+\alpha_{4} A_{V}\right) S_{F}}{N_{H}}=\left(\mu_{1}+\delta_{1}+\gamma\right) I_{F} \\
S_{F}=\frac{N_{H}\left(\mu_{1}+\delta_{1}+\gamma\right) I_{F}}{C_{1} \alpha_{2}(1-\varepsilon \tau) I_{M}+\alpha_{4} A_{V}}
\end{gathered}
$$

To solve for the number of infected female $I_{F}$ at the endemic equilibrium state, we solve 


\section{Stabiizing The Steady State Solution of Lasser Fever: Problems and Prospect}

Equation (3.3)

$$
\begin{aligned}
\frac{d S_{F}}{d T}= & \beta_{H}(1-\theta) N_{H}+\gamma I_{F}-\frac{\left(C_{1} \alpha_{2}(1-\xi j \tau) I_{M}+\alpha_{4} A_{V}\right) S_{F}}{N_{H}}-\mu_{1} S_{F} \\
0= & \beta_{H}(1-\theta) N_{H}+\gamma I_{F}-\frac{\left(C_{1} \alpha_{2}(1-\epsilon \tau) I_{M}+\alpha_{4} A_{V}\right) S_{F}}{N_{H}}-\mu_{1} S_{F} \\
& S_{F}\left[\frac{C_{1} \alpha_{2}(1-\varepsilon \tau) I_{M}+\alpha_{4} A_{V}}{N_{H}}+\mu_{1}\right]=\beta_{H}(1-\theta) N_{H}+\gamma I_{F} \\
S_{F}= & \frac{N_{H}\left[\beta_{H}(1-\theta) N_{H}+\gamma I_{F}\right]}{C_{1} \alpha_{2}(1-\varepsilon \tau) I_{M}+\alpha_{4} A_{V}+N_{H} \mu_{1}}
\end{aligned}
$$

Substituting equation (3.16) into (3.17)

$$
\begin{gathered}
\frac{N_{H}\left(\mu_{1}+\delta_{1}+\gamma\right) I_{F}}{C_{1} \alpha_{2}(1-\varepsilon \tau) I_{M}+\alpha_{4} A_{V}}=\frac{N_{H}\left[\beta_{H}(1-\theta) N_{H}+\gamma I_{F}\right]}{C_{1} \alpha_{2}(1-\varepsilon \tau) I_{M}+\alpha_{4} A_{V}+N_{H} \mu_{1}} \\
\frac{\left(\mu_{1}+\delta_{1}+\gamma\right) I_{F}}{C_{1} \alpha_{2}(1-\xi j \tau) I_{M}+\alpha_{4} A_{V}}=\frac{\left[\beta_{H}(1-\theta) N_{H}+\gamma I_{F}\right]}{C_{1} \alpha_{2}(1-\xi j \tau) I_{M}+\alpha_{4} A_{V}+N_{H} \mu_{1}}
\end{gathered}
$$

Let $C_{2} \alpha_{2}(1-\varepsilon \tau)=q$ and let $\left(\mu_{1}+\delta_{1}+\gamma\right)=A$

Thus

Collect like terms

$$
\begin{gathered}
\frac{A I_{F}}{q I_{M}+\alpha_{4} A_{V}}=\frac{\beta_{H}(1-\theta) N_{H}+\gamma I_{F}}{q I_{M}+\alpha_{4} A_{V}+N_{H} \mu_{1}} \\
A I_{F}\left(q I_{M}+\alpha_{4} A_{V}+N_{H} \mu_{1}\right)=\left(q I_{M}+\alpha_{4} A_{V H}\right)\left(\beta_{H}(1-\theta) N_{H}+\gamma I_{F}\right) \\
A I_{F}\left(q I_{M}+\alpha_{4} A_{V}+N_{H} \mu_{1}\right)=q I_{M} \beta_{H}(1-\theta) N_{H}+q I_{M} \gamma I_{F}+\alpha_{4} A_{V} \beta_{H}(1-\theta) N_{H}+\alpha_{4} A_{V} \gamma I_{F} \\
A I_{F}\left(q I_{M}+\alpha_{4} A_{V}+N_{H} \mu_{1}\right)=\beta_{H}(1-\theta) N_{H}\left(q I_{M}+\alpha_{4} A_{V}\right)+I_{F}\left(q I_{M} \gamma+\alpha_{4} A_{V} \gamma\right)
\end{gathered}
$$

$$
\begin{gathered}
I_{F}\left(q I_{M}+\alpha_{4} A_{V}+N_{H} \mu_{1}\right) A-I_{F}\left(q I_{M} \gamma+\alpha_{4} A_{V} \gamma\right)=\beta_{H}(1-\theta) N_{H}\left(q I_{M}+\alpha_{4} A_{V}\right) \\
I_{F}\left[\left(q I_{M}+\alpha_{4} A_{V}+N_{H} \mu_{1}\right) A-\left(q I_{M} \gamma+\alpha_{4} A_{V} \gamma\right)\right]=\beta_{H}(1-\theta) N_{H}\left(q I_{M}+\alpha_{4} A_{V}\right) \\
I_{F}=\frac{\beta_{H}(1-\theta) N_{H}\left(q I_{M}+\alpha_{4} A_{V}\right)}{\left(q I_{M}+\alpha_{4} A_{V}+N_{H} \mu_{1}\right) A-\left(q I_{M} \gamma+\alpha_{4} A_{V} \gamma\right)}
\end{gathered}
$$

Thus

$$
I_{F}=\frac{\beta_{H}(1-\theta) N_{H}\left(C_{1} \alpha_{2}(1-\varepsilon \tau) I_{M}+\alpha_{4} A_{V}\right)}{\left[C_{1} \alpha_{2}(1-\varepsilon \tau) I_{M}+\alpha_{4} A_{V}+N_{H} \mu_{1}\right]\left(\mu_{1}+\delta_{1}+\gamma\right)-\left(C_{1} \alpha_{2}(1-\varepsilon \tau) I_{M} \gamma+\alpha_{4} A_{V} \gamma\right)}
$$

Similarly for the vector population, we solve equation (5) and (6)

From equation (5)

Also from equation (6)

$$
\begin{gathered}
\frac{d D_{V}}{d T}=\beta_{V} N_{V}-\left(\sigma+\mu_{2}+\delta_{2}\right) D_{V} \\
0=\beta_{V} N_{V}-\left(\sigma+\mu_{2}+\&\right) D_{V} \\
\left(\sigma+\mu_{2}+\&\right) D_{V}=\beta_{V} N_{V} \\
D_{V}=\frac{\beta_{V} N_{V}}{\sigma+\mu_{2}+\delta_{2}}
\end{gathered}
$$

$$
\begin{gathered}
\frac{d A_{V}}{d T}=\sigma D_{V}-\left(\mu_{2}+\&\right) A_{V} \\
0=\sigma D_{V}-\left(\mu_{2}+\delta_{2}\right) A_{V} \\
\sigma D_{V}=\left(\mu_{2}+\delta_{2}\right) A_{V}
\end{gathered}
$$

$A_{V}=\frac{\sigma D_{V}}{\mu_{2}+\delta_{2}}$

So the endemic equilibrium state is given by

$$
\begin{aligned}
& E^{0}=(X, \mathrm{Y}, Z, U, V, W)=\frac{N_{H}\left(\mu_{1}+\delta_{1}+\gamma\right) I_{M}}{C_{2} \alpha_{1}(1-\varepsilon \tau) I_{F}+\alpha_{3} A_{V}}, \\
& \frac{\beta_{H} \theta N_{H}\left[C_{2} \alpha_{1}(1-\varepsilon \tau) I_{F}+\alpha_{3} A_{V}\right]}{\left(\mu_{1}+\delta_{1}+\gamma\right)\left[C_{2} \alpha_{1}(1-\varepsilon \tau) I_{F}+\alpha_{3} A_{V}+N_{H} \mu_{1}\right]-C_{2} \alpha_{1}(1-\varepsilon \tau) I_{F} \gamma-\alpha_{3} A_{V} \gamma}, \\
& \frac{N_{H}\left(\mu_{1}+\delta_{1}+\gamma\right) I_{F}}{C_{1} \alpha_{2}(1-\varepsilon \tau) I_{M}+\alpha_{4} A_{V}}, \\
& \frac{\beta_{H}(1-\theta) N_{H}\left(C_{1} \alpha_{2}(1-\varepsilon \tau) I_{M}+\alpha_{4} A_{V}\right)}{\left[C_{1} \alpha_{2}(1-\varepsilon \tau) I_{M}+\alpha_{4} A_{V}+N_{H} \mu_{1}\right]\left(\mu_{1}+\delta_{1}+\gamma\right)-\left(C_{1} \alpha_{2}(1-\varepsilon \tau) I_{M} \gamma+\alpha_{4} A_{V} \gamma\right)^{\prime}}, \\
& \frac{\beta_{V} N_{V}}{\sigma+\mu_{2}+\delta_{2}}, \\
& \frac{\sigma D_{V}}{\mu_{2}+\delta_{2}}
\end{aligned}
$$




\section{Stabiizing The Steady State Solution of Lasser Fever: Problems and Prospect}

\section{STABILITY ANALYSIS FOR THE EQUILIBRIUM STATE}

We refer to an equilibrium as being stable if the real parts of the eigenvalues of the Jacobian at the equilibrium are negative. An equilibrium is unstable if at least one of the eigenvalues of the Jacobian at that point is positive. In other to obtain the Eigenvalues to check for the stability of the system, we carryout standard linearization of the system by letting the functions $F_{1}, F_{2}, F_{3}, F_{4}, F_{5}$, $F_{6}$ to represent equations (1) $-(6)$ respectively as follows

$$
\begin{aligned}
& F_{1}=\beta_{H} \theta N_{H}+\gamma I_{M}-\frac{\left(C_{2} \alpha_{1}(1-\varepsilon \tau) I_{F}+\alpha_{3} A_{V}\right) S_{M}}{N_{H}}-\mu_{1} S_{M} \\
& F_{2}=\frac{\left(C_{2} \alpha_{1}(1-\varepsilon \tau) I_{F}+\alpha_{3} A_{V}\right) S_{M}}{N_{H}}-\left(\mu_{1}+\delta_{1}+\gamma\right) I_{M} \\
& F_{3}=\beta_{H}(1-\theta) N_{H}+\gamma I_{F}-\frac{\left(C_{1} \alpha_{2}(1-\varepsilon \tau) I_{M}+\alpha_{4} A_{V}\right) S_{F}}{N_{H}}-\mu_{1} S_{F} \\
& F_{4}=\frac{\left(C_{1} \alpha_{2}(1-\varepsilon \tau) I_{M}+\alpha_{4} A_{V}\right) S_{F}}{N_{H}}-\left(\mu_{1}+\delta_{1}+\gamma\right) I_{F} \\
& F_{5}=\beta_{V} N_{V}-\left(\sigma+\mu_{2}+\delta_{2}\right) D_{V} \\
& F_{6}=\sigma D_{V}-\left(\mu_{2}+\delta_{2}\right) A_{V}
\end{aligned}
$$

We differentiate equations (3.27) - (3.32) partially with respect to $S_{M}, I_{M}, S_{F}, I_{F}, D_{V}$ and $A_{V}$ and also putting it in mind that at disease free equilibrium,

Hence;

$$
I_{M}=I_{F}=D_{V}=A_{V}=0
$$

$$
\begin{aligned}
& J_{11}=\frac{\partial F_{1}}{\partial S_{M}}=-\frac{\left(C_{2} \alpha_{1}(1-\varepsilon \tau) I_{F}+\alpha_{3} A_{V}\right)}{N_{H}}-\mu_{1} \\
& J_{21}=\frac{\partial F_{2}}{\partial S_{M}}=\frac{\left(C_{2} \alpha_{1}(1-\varepsilon \tau) I_{F}+\alpha_{3} A_{V}\right)}{N_{H}} \\
& J_{11}\left(I_{F}=0, A_{V}=0\right)=\frac{\partial F_{1}}{\partial S_{M}}=-\mu_{1} \\
& J_{21}\left(I_{F}=0, A_{V}=0\right)=\frac{\partial F_{2}}{\partial S_{M}}=0 \\
& J_{31}=\frac{\partial F_{3}}{\partial S_{M}}=0 \quad J_{41}=\frac{\partial F_{4}}{\partial S_{M}}=0 \\
& J_{51}=\frac{\partial F_{5}}{\partial S_{M}}=0 \quad J_{61}=\frac{\partial F_{6}}{\partial S_{M}}=0 \\
& J_{12}=\frac{\partial F_{1}}{\partial I_{M}}=\gamma \\
& J_{22}=\frac{\partial F_{2}}{\partial I_{M}}=-\left(\mu_{1}+\delta_{1}+\gamma\right)=-A_{1} \\
& J_{32}=\frac{\partial F_{3}}{\partial I_{M}}=-\frac{C_{1} \alpha_{2}(1-\varepsilon \tau) S_{F}}{N_{H}}=-\frac{q Z}{N_{H}} \\
& J_{52}=\frac{\partial F_{5}}{\partial I_{M}}=0 \\
& J_{42}=\frac{\partial F_{4}}{\partial I_{M}}=\frac{C_{2} \alpha_{2}(1-\varepsilon \tau) S_{F}}{N_{H}}=\frac{q Z}{N_{H}} \\
& J_{62}=\frac{\partial F_{6}}{\partial I_{M}}=0 \\
& J_{13}=\frac{\partial F_{1}}{\partial S_{F}}=0 \\
& J_{23}=\frac{\partial F_{2}}{\partial S_{F}}=0 \\
& J_{33}=\frac{\partial F_{3}}{\partial S_{F}}=-\frac{\left(C_{2} \alpha_{1}(1-\varepsilon \tau) I_{M}+\alpha_{4} A_{V}\right)}{N_{H}}-\mu_{1} \\
& J_{43}=\frac{\partial F_{4}}{\partial S_{F}}=\frac{\left(C_{2} \alpha_{1}(1-\varepsilon \tau) I_{M}+\alpha_{4} A_{V}\right)}{N_{H}} \\
& J_{33}\left(I_{M}=0, A_{V}=0\right)=\frac{\partial F_{3}}{\partial S_{F}}=-\mu_{1} \\
& J_{43}\left(I_{M}=0, A_{V}=0\right)=\frac{\partial F_{4}}{\partial S_{F}}=0 \\
& J_{53}=\frac{\partial F_{5}}{\partial S_{F}}=0 \\
& J_{63}=\frac{\partial F_{6}}{\partial S_{F}}=0 \\
& J_{14}=\frac{\partial F_{1}}{\partial I_{F}}=-\frac{C_{2} \alpha_{1}(1-\varepsilon \tau) S_{M}}{N_{H}}=-\frac{p X}{N_{H}} \\
& J_{34}=\frac{\partial F_{3}}{\partial I_{F}}=\gamma \\
& J_{24}=\frac{\partial F_{2}}{\partial I_{F}}=\frac{C_{2} \alpha_{1}(1-\varepsilon \tau) S_{M}}{N_{H}}=\frac{p X}{N_{H}} \\
& J_{54}=\frac{\partial F_{5}}{\partial I_{F}}=0 \\
& J_{44}=\frac{\partial F_{4}}{\partial I_{F}}=-\left(\mu_{1}+\delta_{1}+\gamma\right)=-A_{1} \\
& J_{64}=\frac{\partial F_{6}}{\partial I_{F}}=0 \\
& J_{15}=\frac{\partial F_{1}}{\partial D_{V}}=0 \\
& J_{25}=\frac{\partial F_{2}}{\partial D_{V}}=0 \quad J_{35}=\frac{\partial F_{3}}{\partial D_{V}}=0 \\
& J_{45}=\frac{\partial F_{4}}{\partial D_{V}}=0 \\
& J_{55}=\frac{\partial F_{5}}{\partial D_{V}}=-\left(\sigma+\mu_{2}+\delta_{2}\right)=-A_{2} \quad J_{65}=\frac{\partial F_{6}}{\partial D_{V}}=\sigma \\
& J_{16}=\frac{\partial F_{1}}{\partial A_{V}}=\frac{-\alpha_{3} S_{M}}{N_{H}}=\frac{-\alpha_{3} X}{N_{H}} \quad J_{26}=\frac{\partial F_{2}}{\partial A_{V}}=\frac{\alpha_{3} S_{M}}{N_{H}}=\frac{\alpha_{3} X}{N_{H}} \\
& J_{36}=\frac{\partial F_{3}}{\partial A_{V}}=\frac{-\alpha_{4} S_{F}}{N_{H}}=\frac{-\alpha_{4} Z}{N_{H}} \quad J_{46}=\frac{\partial F_{4}}{\partial A_{V}}=\frac{\alpha_{4} S_{F}}{N_{H}}=\frac{\alpha_{4} Z}{N_{H}} \quad J_{56}=\frac{\partial F_{5}}{\partial A_{V}}=0 \\
& J_{66}=\frac{\partial F_{6}}{\partial A_{V}}=-\left(\mu_{2}+\delta_{2}\right) \\
& \text { Where }(X, \mathrm{Y}, Z, U, V, W)=\left(S_{M}, I_{M}, S_{F}, I_{F}, D_{V}, A_{V}\right) \\
& \begin{aligned}
p & =C_{2} \alpha_{1}(1-\varepsilon \tau) & q & =C_{1} \alpha_{2}(1-\varepsilon \tau) \\
A_{1} & =\left(\mu_{1}+\delta_{1}+\gamma\right) & A_{2} & =\left(\sigma+\mu_{2}+\delta_{2}\right)
\end{aligned}
\end{aligned}
$$


At the DFE, the Jacobian matrix is;

$$
J_{E_{0}}=\left[\begin{array}{cccccc}
-\mu_{1} & \gamma & 0 & -\frac{p x}{N_{H}} & 0 & -\frac{\alpha_{3} x}{N_{H}} \\
0 & -A_{1} & 0 & \frac{p x}{N_{H}} & 0 & \frac{\alpha_{3} x}{N_{H}} \\
0 & -\frac{q z}{N_{H}} & -\mu_{1} & \gamma & 0 & -\frac{\alpha_{4} Z}{N_{H}} \\
0 & \frac{q z}{N_{H}} & 0 & -A_{1} & 0 & \frac{\alpha_{4} Z}{N_{H}} \\
0 & 0 & 0 & 0 & -A_{2} & 0 \\
0 & 0 & 0 & 0 & \sigma & -\left(\mu_{2}+\delta_{2}\right)
\end{array}\right]
$$

We transform (3.33) represented above to an upper triangular matrix using elementary row reduction method to have

$$
\begin{aligned}
& R_{3} \rightarrow R_{3}+R_{4}=\left[\begin{array}{cccccc}
-\mu_{1} & \gamma & 0 & -\frac{p x}{N_{H}} & 0 & -\frac{\alpha_{3} x}{N_{H}} \\
0 & -A_{1} & 0 & \frac{p x}{N_{H}} & 0 & \frac{\alpha_{3} x}{N_{H}} \\
0 & -\frac{q z}{N_{H}} & -\mu_{1} & \gamma & 0 & -\frac{\alpha_{4} z}{N_{H}} \\
0 & \frac{q z}{N_{H}} & 0 & -A_{1} & 0 & \frac{\alpha_{4} z}{N_{H}} \\
0 & 0 & 0 & 0 & -A_{2} & 0 \\
0 & 0 & 0 & 0 & \sigma & -\left(\mu_{2}+\delta_{2}\right)
\end{array}\right] \\
& R_{4} \rightarrow A_{1} R_{4}+\frac{q z}{N_{H}} R_{2}=\left[\begin{array}{cccccc}
-\mu_{1} & \gamma & 0 & -\frac{p x}{N_{H}} & 0 & -\frac{\alpha_{3} x}{N_{H}} \\
0 & -A_{1} & 0 & \frac{p x}{N_{H}} & 0 & \frac{\alpha_{3} x}{N_{H}} \\
0 & 0 & -\mu_{1} & \gamma-A_{1} & 0 & 0 \\
0 & \frac{q z}{N_{H}} & 0 & -A_{1} & 0 & \frac{\alpha_{4} z}{N_{H}} \\
0 & 0 & 0 & 0 & -A_{2} & 0 \\
0 & 0 & 0 & 0 & \sigma & -\left(\mu_{2}+\delta_{2}\right)
\end{array}\right] \\
& R_{4} \rightarrow \frac{1}{A_{1}} R_{4}=\left[\begin{array}{cccccc}
-\mu_{1} & \gamma & 0 & -\frac{p x}{N_{H}} & 0 & -\frac{\alpha_{3} x}{N_{H}} \\
0 & -A_{1} & 0 & \frac{p x}{N_{H}} & 0 & \frac{\alpha_{3} x}{N_{H}} \\
0 & 0 & -\mu_{1} & \gamma-A_{1} & 0 & 0 \\
0 & 0 & 0 & -A_{1}^{2}+\frac{q z p x}{\left(N_{H}\right)^{2}} & 0 & \frac{A_{1} \alpha_{4} z}{N_{H}}+\frac{q z \alpha_{3} x}{\left(N_{H}\right)^{2}} \\
0 & 0 & 0 & 0 & -A_{2} & 0 \\
0 & 0 & 0 & 0 & \sigma & -\left(\mu_{2}+\delta_{2}\right)
\end{array}\right] \\
& R_{6} \rightarrow A_{2} R_{6}+\sigma R_{5}=\left[\begin{array}{cccccc}
-\mu_{1} & \gamma & 0 & -\frac{p x}{N_{H}} & 0 & -\frac{\alpha_{3} x}{N_{H}} \\
0 & -A_{1} & 0 & \frac{p x}{N_{H}} & 0 & \frac{\alpha_{3} x}{N_{H}} \\
0 & 0 & -\mu_{1} & \gamma-A_{1} & 0 & 0 \\
0 & 0 & 0 & -A_{1}+\frac{q z p x}{A_{1}\left(N_{H}\right)^{2}} & 0 & \frac{\alpha_{4} z}{N_{H}}+\frac{q z \alpha_{3} x}{A_{1}\left(N_{H}\right)^{2}} \\
0 & 0 & 0 & 0 & -A_{2} & 0 \\
0 & 0 & 0 & 0 & \sigma & -\left(\mu_{2}+\delta_{2}\right)
\end{array}\right] \\
& {\left[\begin{array}{cccccc}
-\mu_{1} & \gamma & 0 & -\frac{p x}{N_{H}} & 0 & -\frac{\alpha_{3} x}{N_{H}} \\
0 & -A_{1} & 0 & \frac{p x}{N_{H}} & 0 & \frac{\alpha_{3} x}{N_{H}} \\
0 & 0 & -\mu_{1} & \gamma-A_{1} & 0 & 0 \\
0 & 0 & 0 & -A_{1}+\frac{q z p x}{A_{1}\left(N_{H}\right)^{2}} & 0 & \frac{\alpha_{4} z}{N_{H}}+\frac{q z \alpha_{3} x}{A_{1}\left(N_{H}\right)^{2}} \\
0 & 0 & 0 & 0 & -A_{2} & 0 \\
0 & 0 & 0 & 0 & 0 & -A_{2}\left(\mu_{2}+\delta_{2}\right)
\end{array}\right]}
\end{aligned}
$$
Thus the characteristics equation of the upper triangular matrix (3.38) is given by $\mid J_{E_{0}}-\lambda I$

$$
J_{E_{0}}=\left[\begin{array}{cccccc}
-\left(\mu_{1}+\lambda_{1}\right) & \gamma & 0 & -\frac{p x}{N_{H}} & 0 & -\frac{\alpha_{3} x}{N_{H}} \\
0 & -\left(A_{1}+\lambda_{2}\right) & 0 & \frac{p x}{N_{H}} & 0 & \frac{\alpha_{3} x}{N_{H}} \\
0 & 0 & -\left(\mu_{1}+\lambda_{3}\right) & \gamma-A_{1} & 0 & 0 \\
0 & 0 & 0 & -\left(A_{1}-A_{3}+\lambda_{4}\right) & 0 & A_{4} \\
0 & 0 & 0 & 0 & -\left(A_{2}+\lambda_{5}\right) & 0 \\
0 & 0 & 0 & 0 & 0 & -A_{2}\left(\mu_{2}+\delta_{2}\right)-\lambda_{6}
\end{array}\right]
$$

(3.39) 


\section{Stabiizing The Steady State Solution of Lasser Fever: Problems and Prospect}

Equating the product of diagonal of an upper triangular Jacobian matrix to zero (0) gives the eigenvalues of the matrix. Therefore the eigenvalues are

$$
\begin{array}{cc}
-\left(\mu_{1}+\lambda_{1}\right)=0 & -\left(A_{1}+\lambda_{2}\right)=0 \\
\Rightarrow-\mu_{1}-\lambda_{1}=0 & -A_{1}-\lambda_{2}=0 \\
\Rightarrow \lambda_{1}=-\mu_{1} & \Rightarrow \lambda_{2}=-A_{1} \\
-\left(A_{1}-A_{3}+\lambda_{4}\right)=0 & -\left(A_{2}+\lambda_{5}\right)=0 \\
-\left(A_{1}-A_{3}\right)-\lambda_{4}=0 & -A_{2}-\lambda_{5}=0 \\
\Rightarrow \lambda_{4}=-\left(A_{1}-A_{3}\right) & \Rightarrow \lambda_{5}=-A_{2}
\end{array}
$$

$$
\begin{gathered}
-\left(\mu_{1}+\lambda_{3}\right)=0 \\
-\mu_{1}-\lambda_{3}=0 \\
\Rightarrow \lambda_{3}=-\mu_{1} \\
-A_{2}\left(\mu_{2}+\delta_{2}\right)-\lambda_{6}=0 \\
-A_{2}\left(\mu_{2}+\delta_{2}\right)=\lambda_{6} \\
\Rightarrow \lambda_{6}=-A_{2}\left(\mu_{2}+\delta_{2}\right)
\end{gathered}
$$

Where identity matrix $(\mathrm{I})$ is

$$
I=\left\{\begin{array}{llllll}
1 & 0 & 0 & 0 & 0 & 0 \\
0 & 1 & 0 & 0 & 0 & 0 \\
0 & 0 & 1 & 0 & 0 & 0 \\
0 & 0 & 0 & 1 & 0 & 0 \\
0 & 0 & 0 & 0 & 1 & 0 \\
0 & 0 & 0 & 0 & 0 & 1
\end{array}\right\}
$$

Thus;

$$
\begin{gathered}
|\lambda I|=\left\{\begin{array}{cccccc}
\lambda_{1} & 0 & 0 & 0 & 0 & 0 \\
0 & \lambda_{2} & 0 & 0 & 0 & 0 \\
0 & 0 & \lambda_{3} & 0 & 0 & 0 \\
0 & 0 & 0 & \lambda_{4} & 0 & 0 \\
0 & 0 & 0 & 0 & \lambda_{5} & 0 \\
0 & 0 & 0 & 0 & 0 & \lambda_{6}
\end{array}\right\} \\
A_{1}=\left(\mu_{1}+\delta_{1}+\gamma\right) \\
A_{3}=\frac{q Z p X}{A_{1}\left(N_{H}\right)^{2}}
\end{gathered}
$$

Hence

$$
\begin{array}{cc}
\lambda_{1}=-\mu_{1} \quad \lambda_{2}=-\left(\mu_{1}+\delta_{1}+\gamma\right) & \lambda_{3}=-\mu_{1} \\
\lambda_{4}=-\left[\left(\mu_{1}+\delta_{1}+\gamma\right)-\frac{q Z p X}{\left(\mu_{1}+\delta_{1}+\gamma\right)\left(N_{H}\right)^{2}}\right] & \lambda_{5}=-\left(\sigma+\mu_{2}+\delta_{2}\right)
\end{array}
$$

$\lambda_{6}=-\left(\sigma+\mu_{2}+\delta_{2}\right)\left(\mu_{2}+\delta_{2}\right)$

For stability of disease-free equilibrium Routh-Hurwitz criteria requires that all eigenvalues have negative real part. Since all the eigenvalues of (3.39) have negative real parts i.e. $\lambda_{i}<0$ for

$\mathrm{i}=1,2,3, \ldots, 6$ implies that the disease free equilibrium is stable. Thus the disease dies out in the long run and cannot invade the population.

If $R_{e}(\lambda)>0$, the disease free equilibrium is unstable i.e. invasion is always possible and the infection will be able to spread in the population.

An endemic disease is a disease that is always present in a certain population or region. In DFE, we solve to know if the disease cannot invade a population or can but DEE, we already know that the disease have invaded the population and it is persistence. Once the disease invades the population, it becomes endemic.

\section{CONCLUSION}

The study analysed the spread and control and control of Lassa fever. Lassa fever is an acute viral haemorrhagic illness caused by Lassa virus. Noting that humans usually become infected with Lassa virus through exposure to food or household items contaminated with urine or feaces of infected Mastomys rats.

The stability analysis for disease free equilibrium of our model showed that the disease dies out in the long run and cannot invade the population.

\section{REFERENCES}

1) Onuorah, M. O., Akinwande, N. I., Nasir, M. O., \& Ojo, M. S. (2016). Sensitivity analysis of Lassa fever model. International J. of Mathematics and Statistic Studies, 4, 30-49.

2) Onuorah, M. O., Ojo, M. S., Usman, D. J., \& Ademu, A. (2016). Basic reproductive Number for the spread and control of Lassa Fever. International Journal of Mathematics Trends and Technology (IJMTT), 30(1), 1-7.

3) Usman, S., \& Adamu, I. I. (2018). Modelling the Transmission Dynamics of the Lassa Fever Infection. Mathematical Theory 


\section{Stabiizing The Steady State Solution of Lasser Fever: Problems and Prospect}

and Modeling, 8(5), 42-63.

4) Eraikhuemen, B. I., \& Eguasa, O. (2017). Lassa fever and its Control Measures, J. of Natural Sc. Research, 7, 12.

5) Faniran, T. S. (2017). A Mathematical Modelling of Lassa Fever Dynamics with Non-drug Compliance Rate. Int J. Math. Trends Technol, 47(5), 305-317.

6) Onuorah, M. O., Akinwande, N. I., Nasir, M. O., \& Ojo, M. S. (2016). Sensitivity analysis of Lassa fever model. International J. of Mathematics and Statistic Studies, 4, 30-49.

7) AKINADE, M. O., \& AFOLABI, A. S. (2020). Sensitivity And Stability Analyses Of A Lassa Fever Disease Model With Control Strategies. IOSR Journal of Mathematics (IOSR-JM), 16(1), 29-42.

8) Onah, I. S., Adewoye, R. A., \& Mbah, G. C. E. (2019). Sensitivity Analysis of Multiple Control Intervention Measures of Lassa fever Disease Model. International Journal of Mathematics Trends and Technology (IJMTT) - Vol. 65.

9) Omoloye, M. A., Yusuff, M. I., \& Emiola, O. K. S. (2020). Application of differential transformation method for solving dynamical transmission of Lassa fever model. World Academy of Science, Engineering and Technology International Journal of Physical and Mathematical Sciences Vol, 14, 151-154.

10) Richmond, J. K., \& Baglole, D. J. (2003). Lassa fever: epidemiology, clinical features, and social consequences. Bmj, 327(7426), 1271-1275.

11) Trottier, H., \& Phillipe, P. (2001). "Deterministic modeling of infectious diseases: theory and methods". The Internet Journal of Infectious Diseases. 